\title{
Complications of Pelvic Exenteration Encountered by both Surgical Oncologists and Anesthesiologists
}

\author{
Ethem Unal1* , Abdullah Yildiz¹, Sema Yuksekdag ${ }^{1}$ and Aysun Firat ${ }^{2}$ \\ ${ }^{1}$ Department of General Surgery, Health Sciences University, Umraniye Education and \\ Research Hospital, Istanbul, Turkey \\ 2Department of Obstetrics and Gynaecology, Health Sciences University, Istanbul \\ Education and Research Hospital, Istanbul, Turkey
}

Mini Review

Volume 4 Issue 3

Received Date: September 24, 2019

Published Date: October 03, 2019

DOI: $10.23880 /$ accmj-16000159

*Corresponding author: Ethem Unal, MD, PhD, Associate Professor of Surgery and Surgical Oncology, Health Sciences University, Umraniye Education and Research Hospital, Istanbul, Turkey, Tel: +90 532258 4917; Email: drethemunal@gmail.com

\section{Introduction}

Although Pelvic Exenteration (PE) was seen as one of the most destructive surgical procedures associated with unacceptable rates of perioperative morbidity and mortality, currently, this perception has changed and these operations are gaining popularity again all over the world [1]. Advanced primary or recurrent pelvic malignancies, gynecologic tumors (cervix, endometrium, over, vulva and vagina), urologic (bladder, prostate) and rectal cancers are candidates for PE. It has also been used for palliation of symptoms related to radiation necrosis or extensive tumor burden. First series of PE performed and reported by Brunschwig included 22 cases with 5 died during early postoperative period [2]. Since then, improvements in critical care, use of antibiotics, hyper alimentation and prophylaxis for thromboembolism, advances in surgical instruments such as staplers and in surgical techniques, including urinary conduits and pelvic reconstruction, have improved morbidity and mortality. However, reconstruction procedures may also create new problems, as well. Recent rates of morbidity and perioperative mortality are $30-60 \%$ and $3-5 \%$, respectively [3-5]. In fact, these ratios are still high. Furthermore, there are reports presenting higher ratios of morbidity, up to $90 \%$ [6]. Intensive care units (ICU) with adequate equipments and health care takers can make it easier to choose candidates for operation, but the numbers and rates mentioned above should alert both anesthesiologists and surgeons to take immediate steps.

Postoperative complications of a PE will depend mainly on what was removed and the patient's overall health condition. In general, the most common major complications are intraabdominal collections and wound infections [3,7]. Major complications of operative field involve gastrointestinal tract (fistula or obstruction), urinary tract (fistula, infection, or obstruction), or wound (abscess, dehiscence/necrosis, or hemorrhage). Metabolic complications such as hyperchloremic metabolic acidosis can also be seen in patients with urinary conduit.

The absence of well-defined preoperative selection criteria to identify patients eligible for such a complicated operation like PE causes important problems perioperatively. In a recent study, the assessment of preoperative predictors of 30-day major surgical complications has been prompted in patients undergoing PE. In this study, severe postoperative complications (urinary reconstruction, wound dehiscence and abdominal abscess) were seen in $27 \%$ of the patients [7]. Besides, $2.2 \%$ of patients died in 3 months. The authors stated that independent predictors of severe postoperative complications were anterior or total PE, 


\section{Anaesthesia \& Critical Care Medicine Journal}

pre-operative hemoglobin $\leq 10 \mathrm{mg} / \mathrm{dl}$ and presence of $3+$ comorbidities. In an another retrospective study in large series with PE, 49\% of patients developed major complications of the operative field (gut, urinary tract and wound itself), and operative mortality was $2.9 \%$ [8]. It has also been showed that palbumin levels less than 3.5 $\mathrm{g} / \mathrm{dL}$ and history of deep vein thrombosis were associated with higher morbidity [5]. Low albumin levels independently correlated with readmission. Postoperative complications and readmission increase the cost, as well.

In conclusion, preoperative patient selection is the key point for the reduction of postoperative complications after PE. Therefore, it is clear that surgeons and anesthesiologists should work together, especially in the preoperative period, to have better results.

\section{References}

1. Bacalbasa N, Balescu I (2015) Pelvic exenterationreconsidering the procedure. J Med Life 8(2): 146149.

2. Brunschwig A (1943) Complete excision of pelvic viscera for advanced carcinoma, Cancer.

3. Peacock O, Waters PS, Kong JC, Warrier SK, Wakeman C, et al. (2019) Complications after extended radical resections for locally advanced and recurrent pelvic malignancies: A 25-year experience. Ann Surg Oncol.

4. Waters PS, Peacock O, Warrier SK, Wakeman C, Eglinton T, et al. (2019) Evolution of pelvic exenteration surgery- resectional trends and survival outcomes over three decades. Eur J Surg Oncol.

5. Bogani G, Signorelli M, Ditto A, Martinelli F, Casarin J, et al. (2018) Factors Predictive of 90-day morbidity, readmission, and costs in patients undergoing pelvic exenteration. Int J Gynecol Cancer 28(5): 975-982.

6. Platt E, Dovell G, Smolarek S (2018) Systematic review of outcomes following pelvic exenteration for the treatment of primary and recurrent locally advanced rectal cancer. Tech Coloproctol 22(11): 835-845.

7. Tortorella L, Casarin J, Mara KC, Weaver AL, Multinu F, et al. (2019) Prediction of short-term surgical complications in women undergoing pelvic exenteration for gynecological malignancies. Gynecol Oncol 152(1): 151-156.

8. Jakowatz JG, Porudominsky D, Riihimaki DU, Kemeny M, Kokal WA, et al. (1985) Complications of Pelvic Exenteration. Arch Surg 120(11): 1261-1265. 